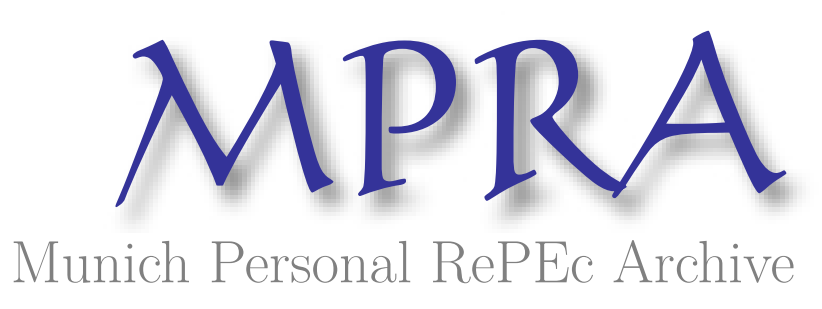

\title{
Dynamic Regimes of a Multi-agent Stock Market Model
}

\author{
Yu, Tongkui and Li, Honggang \\ Department of Systems Science, Beijing Normal University
}

November 2008

Online at https://mpra.ub.uni-muenchen.de/14339/

MPRA Paper No. 14339, posted 30 Mar 2009 01:59 UTC 


\title{
Dynamic Regimes of a Multi-agent Stock Market Model
}

\author{
Tongkui $\mathrm{Yu}$ and Honggang Li \\ Department of Systems Science, Beijing Normal University, \\ Beijing, 100875, P.R.China
}

\begin{abstract}
This paper presents a stochastic multi-agent model of stock market. The market dynamics include switches between chartists and fundamentalists and switches in the prevailing opinions (optimistic or pessimistic) among chartists. A nonlinear dynamical system is derived to depict the underlying mechanisms of market evolvement. Under different settings of parameters representing traders' mimetic contagion propensity, price chasing propensity and strategy switching propensity, the system exhibits four kinds of dynamic regimes: fundamental equilibrium, non-fundamental equilibrium, periodicity and chaos.
\end{abstract}

Key words: multi-agent stock market model, market dynamic regime, bifurcation analysis

\section{Introduction}

Stock market exhibits a variety of dynamic regimes. According to efficient market hypothesis and rational expectation theory, market price always fluctuates around the fundamental values [1]. While in real market the price may deviate from fundamental value persistently, and plenty of empirical work gives the evidence for rational speculative bubbles [2-4]. Market can also be in a periodic regime commonly described as bull and bear cycle [5-7]. And researchers even investigated the chaotic behaviors in stock market [8].

Therefor, modeling the dynamics of financial market has attracted much interest of researchers in recent years. Different models have been presented to describe the market dynamic regimes including equilibrium, periodicity and chaos [9-14].

But less attention has been paid to the influence of traders' behaviors to market dynamic regimes. In fact traders' behavior play a important role in financial market, many traders regard stock market full of speculative opportunities, they use technical strategy to forecast future price, and traders' emotional factors may drive market into bubbles and crashes [15]. There is even a new subject called behavioral finance which studies how behavioral factors affect finance market [16].

Inspired by statistical physics, Lux and Marchesi presented a model with switching mechanism where fundamentalists and chartists may compare their 
performance and switch to each other $[17,18]$. This model is built from the bottom to up: first considering the basic behavioral pattern to model the switch possibility of traders among different groups, then deriving a dynamical system to approximate the market evolution. Hence the dynamical system reserves the traders' main behavioral propensities. Following the line of Lux, we present a stock market model with four kinds of dynamic regimes: fundamental equilibrium, non-fundamental equilibrium, periodicity and chaos, and concentrate on analyzing the effect of traders behavioral propensities such as mimetic propensity, price-chasing propensity and strategy-switching propensity on the market dynamic regimes.

\section{The model}

In this model, $N$ traders deal in a single financial asset with fixed fundamental value $p_{f}$. Three groups of agents are considered. First, $N$ traders are split up into the groups of chartists and fundamentalists with $n_{c}(t)$ and $n_{f}(t)$ denoting the (time-varying) numbers of agents in both groups $\left(n_{c}+n_{f}=N\right)$. Fundamentalists expect the price to follow the fundamental value of the asset and buy (sell) when the actual market price is believed to be below (above) the fundamental value. While chartists attempt to identify price trends and patterns, and consider the behavior of other traders as a source of information, which results in a tendency towards herding behavior. Second, the chartists group consists of optimistic and pessimistic individuals whose numbers are given by $n_{+}(t)$ and $n_{-}(t)$ with $n_{+}(t)+$ $n_{-}(t)=n_{c}$. The optimists will buy additional units of the asset, whereas the pessimists will sell part of their actual holdings of the asset. The major building blocks of the model are transitions of agents from one group to another together with endogenous price changes resulting from the agentsoperations.

\subsection{Market components}

First, the probabilities of switches of agents from the pessimistic to the optimistic subgroup and vice versa are denoted by $\pi_{+-} \Delta t$ and $\pi_{-+} \Delta t$ during a small time increment $\Delta t$, where $\pi_{+-}$and $\pi_{-+}$are concretized as follows:

$$
\pi_{+-}=v_{1} \exp \left(U_{1}\right), \pi_{-+}=v_{1} \exp \left(-U_{1}\right)
$$

with $U_{1}=\alpha_{1} x+\alpha_{2} \dot{p} / v_{1}, x=\left(n_{+}-n_{-}\right) / n_{c}$. Here, the basic influences on the chartists' formation of opinion are the majority opinion of their fellow traders $x$ and the actual price trend, $(d p / d t) / v_{1}$. Parameter $v_{1}$ is the frequency of revaluation of opinion, $\alpha_{1}$, and $\alpha_{2}$ are the measure of the importance of majority opinion and price trend in chartists' behavior respectively, we call them mimetic propensity and price-chasing propensity.

Chartists and fundamentalists are assumed to meet each other at random and possibly change their strategy by comparing the performance. Switching from the chartist to the fundamentalist group and vice versa are formalized 
in a similar manner. The notational convention in the transition probabilities below is also that the first index denotes the subgroup to which a trader moves and the second index gives the subgroup to which he formerly belonged (hence, as an example, $\pi_{+f} \Delta t$ is the probability for a fundamentalist to switch to the optimistic chartists' group):

$$
\begin{aligned}
& \pi_{+f}=v_{2} \exp \left(U_{2,1}\right), \pi_{f+}=v_{2} \exp \left(-U_{2,1}\right) \\
& \pi_{-f}=v_{2} \exp \left(U_{2,2}\right), \pi_{f-}=v_{2} \exp \left(-U_{2,2}\right)
\end{aligned}
$$

with

$$
\begin{aligned}
U_{2,1} & =\alpha_{3}\left[\left(r+\dot{p} / v_{2}\right) / p-R-s\left|\left(p_{f}-p\right) / p\right|\right] \\
U_{2,2} & =\alpha_{3}\left[R-\left(r+\dot{p} / v_{2}\right) / p-s\left|\left(p_{f}-p\right) / p\right|\right]
\end{aligned}
$$

The forcing terms $U_{2,1}$ and $U_{2,2}$ for these transitions depend on the difference between the profits earned by chartists and fundamentalists. Here $v_{2}$ is the frequency with which agents reconsider their trading strategies, and $\alpha_{3}$ is the sensitivity of agents to profit differentials, called strategy-switching propensity. Profits enjoyed by chartists from the optimistic group are composed of nominal dividends $r$ and capital gains due to the price change $d p / d t$. Dividing by the actual market price gives the revenue per unit of the asset. Excess returns are computed by subtracting the average real risk-adjusted return $R$ available from other investments. Fundamentalists, on the other hand, consider the deviation between price and fundamental value $p_{f}$ (irrespective of its sign) as the source of arbitrage opportunities. As the gains from arbitrage occur only in the future it is discounted by a factor $s<1$. Furthermore, neglecting the dividend term in fundamentalists' profits is justified by assuming that they correctly perceive the (long-term) real returns as equally as the average return of alternative investments, i.e. $R=r / p_{f}$.

Entry and exit are incorporated by assuming that a constant portion $(a)$ of traders is regularly replaced by new entrants. It is natural for this assumption because there always be some investors who need cash for emergent use, and some others who have free money to invest. Both fundamentalists and chartists are equally likely to exit, the newcomers act as chartists and fundamentalists in a fixed proportion, saying $b(b \in[0,1])$ of newcomers as fundamentalists and $1-b$ as chartists. Newcomers of chartists follow the prevailing market opinion(optimistic or pessimistic), that is the distribution of market opinion in new chartists is the same as current market. Exit and entry mechanism can also be described as probability transition. For example, among $n_{f}$ fundamentalists, we have $a n_{f}$ of them exit and $(1-a) n_{f}$ stay. Corresponding to those exited, $a b n_{f}$ fundamentalists and $a(1-b) n_{f}$ chartists including $a(1-b) n_{f}\left(n_{+} / n_{c}\right)$ and $a(1-b) n_{f}\left(n_{-} / n_{c}\right)$ enter. For $(1-a) n_{f}$ fundamentalists left, according to above transition probabilities, $(1-a) n_{f} \pi_{+f}\left(n_{+} / N\right)$ of them switch to optimistic chartists and $(1-a) n_{f} \pi_{-f}\left(n_{-} / N\right)$ switch to pessimistic chartists, leaving $(1-a) n_{f}\left[1-\pi_{+f}\left(n_{+} / N\right)-\pi_{-f}\left(n_{-} / N\right)\right]$ keep as fundamentalists. The transitions of pessimistic and optimistic chartists are similarly available. Figure 1 illustrates the transitions among the three groups. 


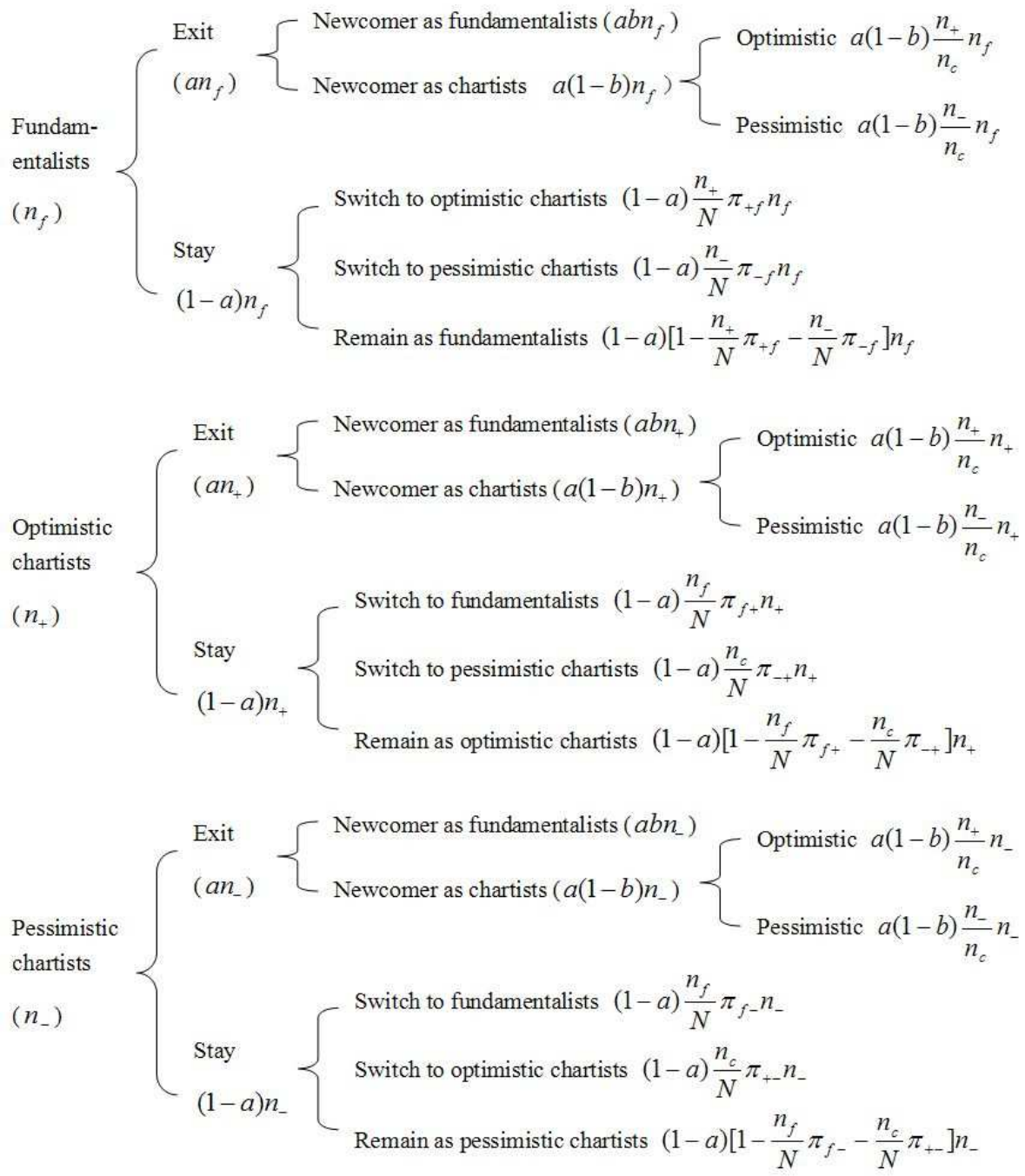

Fig. 1. Transitions among three groups

Lastly, the dynamics of the asset's price results from the market operations of our agents and the ensuing price adjustment by a market maker who reacts sluggishly on excess demand. Assuming a constant average trading volume per transaction $T_{c}$, excess demand of chartists is $E D_{c}=\left(n_{+}-n_{-}\right) T_{c}$. Excess demand of fundamentalists $E D_{f}=n_{f} \gamma\left(p_{f}-p\right) / p$ with $\gamma$ being a parameter for the strength of reaction on price deviation. So, $d p / d t=\beta\left(E D_{c}+E D_{f}\right)$, where $\beta$ denotes the price adjustment strength. 


\subsection{Market dynamics}

In this part,we derive a three dimensional differential equations system to approximate the dynamics of the model above. The three variables used to describe the market states are $x=\left(n_{+}-n_{-}\right) / n_{c}$ (named market confidence index $), y=n_{f} / N$ (named market rationality index) and price $p$. According to the transition mechanism defined above(see figure 1), the change of optimistic chartists number $n_{+}$at a time point is given as:

$$
\begin{aligned}
d n_{+} / d t= & a(1-b) \frac{n_{+}}{n_{c}} n_{f}+(1-a) \frac{n_{+}}{N} \pi_{+f} n_{f}+a(1-b) \frac{n_{+}}{n_{c}} n_{-}+(1-a) \frac{n_{c}}{N} \pi_{+-} n_{-} \\
& -a b n_{+}-a(1-b) \frac{n_{-}}{n_{c}} n_{+}-(1-a) \frac{n_{f}}{N} \pi_{f+} n_{+}-(1-a) \frac{n_{c}}{N} \pi_{-+} n_{+}
\end{aligned}
$$

Rearrange equation (3):

$$
\begin{aligned}
d n_{+} / d t= & (1-a)\left[\left(n_{-} \pi_{+-}-n+\pi_{-+}\right)+\left(n_{f} \pi_{+f}-n_{+} \pi_{f+}\right)\right] \\
& +a(1-a) n_{f} \frac{n_{+}}{n_{c}}-a b n_{+}
\end{aligned}
$$

Similarly, the change of number of pessimistic chartists is:

$$
\begin{aligned}
d n_{-} / d t= & (1-a)\left[\left(n_{+} \pi_{-+}-n_{-} \pi_{+-}\right)+\left(n_{f} \pi_{-f}-n_{-} \pi_{f-}\right)\right] \\
& +a(1-a) n_{f} \frac{n_{-}}{n_{c}}-a b n_{-}
\end{aligned}
$$

From $n_{c}=n_{+}+n_{-}$, one arrives at $d n_{c} / d t=d\left(n_{+}+n_{-}\right) / d t=d n_{+} / d t+$ $d n_{-} / d t$. So the time evolution of $n_{c}$ is:

$$
d n_{c} / d t=(1-a)\left[n_{f} \pi_{+f}-n_{+} \pi_{f+}+n_{f} \pi_{-f}\right]-a(1-b)
$$

As defined, market confidence index $x=\left(n_{+}-n_{-}\right) / n_{c}$, market rationality index $y=n_{f} / N$. Note that $n_{c}=n_{+}+n_{-}, n_{c}+n_{f}=N$. It then follows that $n_{f}=y N, n_{+}=(1+x)(1-y) N$, and $n_{-}=(1-x)(1-y) N$. Following derivative rules, one can get:

$$
\begin{aligned}
d x / d t= & (1-a)(1-y)\left[(1-x) \pi_{+-}-(1+x) \pi_{-+}\right] \\
& +\frac{1}{2} y\left(1-x^{2}\right)\left(\pi_{+f}-\pi_{f+}+\pi_{f-}-\pi_{-f}\right)
\end{aligned}
$$

One may note that the terms from 'entry-exit' mechanism cancel out, which means that they do not influence the distribution of the opinion among chartists. This makes sense for our assumption that the newcomers of chartists follow the prevailing market opinion.

The evolution of $y$ can also be derived as:

$$
\begin{aligned}
d y / d t= & \frac{1}{2}(1-a) y(1-y)(1+x)\left(\pi_{+f}-\pi_{f+}\right) \\
& +\frac{1}{2}(1-a) y(1-y)(1-x)\left(\pi_{-f}-\pi_{f-}\right)+a(1-b) y
\end{aligned}
$$


We can also notice that $(N)$ disappears in $d x / d t$ and $d y / d t$. This means that it is not the number of traders but the distribution among different groups that determines the market dynamics.

According to mode setting above, the change price $d p / d t=\beta\left(E D_{c}+E D_{f}\right)$ can be formulated as a function of our market state variables $x, y$ and $p$.

$$
d p / d t=\beta\left[\beta\left[x(1-y) T_{c}+y\left(p_{f}-p\right) T_{f}\right]\right]
$$

Inserting the transition possibility (1) and (2) into time development of market confidence index (7), market rationality index(8) and price (9), we get:

$$
\left\{\begin{aligned}
\dot{x}= & 2 v_{1}(1-a)(1-y)\left[\tanh \left(U_{1}\right)-x\right] \cosh \left(U_{1}\right) \\
& +v_{2}(1-a)\left(1-x^{2}\right)\left[\sinh \left(U_{2,1}\right)-\sinh \left(U_{2,2}\right)\right] \\
\dot{y}= & -v_{2}(1-a) y(1-y)\left[(1+x) \sinh \left(U_{2,1}\right)\right. \\
& \left.+(1-x) \sinh \left(U_{2,2}\right)\right]-a(1-b) y+a b(1-y) \\
\dot{p}= & \beta\left[x(1-y) T_{c}+y\left(p_{f}-p\right) T_{f}\right]
\end{aligned}\right.
$$

where

$$
\begin{aligned}
& U_{1}=\alpha_{1} x+\alpha_{2} \dot{p} / v_{1} \\
& U_{2,1}=\alpha_{3}\left[\left(r+\dot{p} / v_{2}\right) / p-R-s\left|\left(p_{f}-p\right) / p\right|\right] \\
& U_{2,2}=\alpha_{3}\left[R-\left(r+\dot{p} / v_{2}\right) / p-s\left|\left(p_{f}-p\right) / p\right|\right]
\end{aligned}
$$

This system governs the evolution of the multi-agent stock market model. Using certain approximation schemes [19], it can be shown to coincide with a first-order approximation to the exact dynamics of mean values of the underling stochastic model. When $a=0$ (no 'entry-exit' mechanism), system (10) equals Lux model in [17]. This means that our modified model generalizes it and makes it a special case.

\section{Market dynamic regimes}

In this part, we analyze system (10) with both analytical and numerical approach to find different dynamic regimes. At the same time, we mainly concentrate on understanding how traders behavioral propensities such as mimetic contagion propensity $\left(\alpha_{1}\right)$, price-chasing propensity $\left(\alpha_{2}\right)$ and strategy switching propensity $\left(\alpha_{3}\right)$ influence the market dynamic regimes.

\subsection{Fundamental equilibrium and stability}

Fundamental equilibrium with market price equaling underlying asset value exists in this model. Proposition 1 gives the existence of such an equilibrium.

Proposition 1. For all parameter values, $\left(x^{*}=0, y^{*}=b, p^{*}=p_{f}\right)$ is an equilibrium of system (10). 
Proof. With $x^{*}=0, p^{*}=p_{f}$, one gets $d p / d t=0$.

Then inserting $d p / d t=0, x^{*}=0$ to RHS of $U_{1}$ equation, $U_{1}=0$. Because $p^{*}=p_{f}, r=R p_{f}, x^{*}=0, U_{2,1}=U_{2,2}=0$. Then we can see that $d x / d t=0$.

With $y^{*}=b$, the last two terms of doty equation cancel out. With $U_{2,1}=$ $U_{2,2}=0$, one gets $d y / d t=0$.

Under fundamental equilibrium, the market price equals the underlying value of assets, so there no price-chasing opportunities. The forces of optimistic chartists and pessimistic chartists are in balance, and mimetic effect cancels out. The market rationality index $y=n_{f} / N$ equals the constant $b$, which is the portion of fundamentalists in all the newcomers in the 'entry-exit' mechanism. This means that in such case the 'entry-exit' mechanism has no influence to market dynamics.

Proposition 2 provides the stability conditions of fundamental equilibrium.

Proposition 2. Under the conditions

$$
\begin{aligned}
& \text { (i) } v_{1} \alpha_{1}+(1-b) \beta T_{c} \alpha_{2}+\frac{b \beta T_{c}}{p_{f}} \alpha_{3}<v_{1}+\frac{\beta b T_{f}}{2(1-a)(1-b)} \\
& \text { (ii) } v_{1} p_{f} T_{f} \alpha_{1}-v_{2} T_{c} R \alpha_{3}-v_{1} p_{f} T_{f}<0
\end{aligned}
$$

$\left(x^{*}=0, y^{*}=b, p^{*}=p_{f}\right)$ is a stable equilibrium of system (10).

Proof. The entries of the three-by-three Jacobian $A$ of system (10), evaluated at the equilibrium $\left(x^{*}=0, y^{*}=b, p^{*}=p_{f}\right)$ are:

$$
\begin{aligned}
& \left.a_{11}=\frac{\partial \dot{x}}{\partial x}=2(1-a)(1-b)\left[v_{1} \alpha_{1}+\alpha_{2} \beta T_{c}(1-b)-v_{1}\right)+\alpha_{3} \beta b T_{c} / p_{f}\right], \\
& a_{12}=\frac{\partial \dot{x}}{\partial y}=0, \quad a_{13}=\frac{\partial \dot{x}}{\partial p}=-2(1-a)\left[(1-b) \beta T_{f} \alpha_{2}+\frac{v_{2} r}{\left(p_{f}\right)^{2}}+\frac{\beta b T_{f}}{p_{f}},\right. \\
& a_{21}=\frac{\partial \dot{y}}{\partial x}=0, \quad a_{22}=\frac{\partial \dot{y}}{\partial y}=-a, \\
& a_{23}=\frac{\partial \dot{y}}{\partial p}=\left\{\begin{array}{cc}
-2 v_{2} \alpha_{3} s(1-a) b(1-b) / p_{f} & p<p_{f} \\
2 v_{2} \alpha_{3} s(1-a) b(1-b) / p_{f} & p<p_{f}
\end{array},\right. \\
& a_{31}=\frac{\partial \dot{p}}{\partial x}=\beta(1-b) T_{c}, \quad a_{32}=\frac{\partial \dot{p}}{\partial y}=0, \quad a_{33}=\frac{\partial \dot{p}}{\partial p}=-\beta b T_{f}
\end{aligned}
$$

The discontinuity in the entry $a_{23}$ stems from the absolute term in the profit differential. In the case of continuous derivatives, stability hinges on the signs of the roots of the matrix A. Considering separately the cases $p<p_{f}$ and $p>p_{f}$, one finds that in both cases the roots $\lambda_{i}(i=1,2,3)$ are determined by the same characteristic equation: $\left(a_{22}-\lambda\right)\left[\left(a_{11}-\lambda\right)\left(a_{33}-\lambda\right)-a_{13} a_{31}\right]=0$.

Obviously, $\lambda_{1}=a_{22}=-a<0$ is a eigenvalue of $A$. If the other two eigenvalues are less than $0,\left(x^{*}=0, y^{*}=b, p^{*}=p_{f}\right)$ is a stable equilibrium. This requires the quadratic equation of $\lambda, \lambda^{2}-\left(a_{11}+a_{22}\right) \lambda+a_{11} a_{33}-a_{13} a_{31}=0$ has two negative roots. By Vieta's Theorem, one arrives at $a_{11}+a_{33}<0$ and $a_{11} a_{33}-a_{13} a_{31}>0$. Rearranging these two inequalities gives the sufficient conditions as (11) of local stability of fixed point $\left(x^{*}=0, y^{*}=b, p^{*}=p_{f}\right)$. 
A close look at these conditions gives some insights. Because we focus on analysis of the influence of traders propensities to market dynamics, we keep other parameters fixed, following Lux's setting $v_{1}=0.5, v_{2}=0.75, a=0.01, b=$ $0.05, \beta=0.5, R=0.1, T_{c}=T_{f}=5, p_{f}=10, r=R / p_{f}=0.01$. All the analysis and experiments in this paper are based on this setting. Condition $(i)$ requires that a linear combination of $\alpha_{1}, \alpha_{2}, \alpha_{3}$ must less than a positive value, this implies that the larger those traders behavioral propensities are, the more possible the condition $(i)$ is violated. Investigating the coefficients of them gives that $\alpha_{2}$ is more easier to violate the condition because it multiples with $T_{c}$ which is usually relatively large. Rearranging condition $(i i)$, one can get $\alpha_{1}<\frac{v_{2} T_{c} R}{v_{1} p_{f} T_{f}} \alpha_{3}+1$. This implies that there is a interdependence between mimetic contagion propensity $\alpha_{1}$ and strategy switching propensity $\alpha_{3}$. An upper value for the parameter $\alpha_{1}$ associated with $\alpha_{3}$, saying $\overline{\alpha_{1}}$, exists beyond which the fundamental equilibrium will lose stability.

\subsection{Non-fundamental equilibria}

Non-fundamental equilibria is referred as that the market price deviates from fundamental value persistently. Proposition 3 provides the possible existence of such equilibria.

Proposition 3. (i) For $\alpha_{1} \leq 1$, no equilibria other than the fundamental equilibrium exist in system (10). (ii) For $\alpha_{1}>1$, there may exist additional equilibria $E_{+}\left(x_{+}>0, p_{+}>p_{f}, y_{+}>b\right)$ and $E_{-}\left(x_{-}<0, p_{-}<p_{f}, y_{-}>b\right)$

Proof. From $d p / d t=0$ it can be seen that possible equilibria $x_{+}>0\left(x_{-}<0\right)$ are connected with price $p_{+}>p_{f}\left(p_{-}<p_{f}\right)$. Then $U_{2,1}<0, U_{2,2}>U_{2,1}$ when $x_{+}>0, p_{+}>p_{f}$ and $U_{2,2}<0, U_{2,1}>U_{2,2}$ when $x_{-}<0, p_{-}<p_{f}$. This implies that the second term in the RHS of $d x / d t$ equation is negative for any pair $\left(x_{+}, p_{+}\right)$and positive for any $\left(x_{-}, p_{-}\right)$. Hence in order to obtain stability, the first term has to carry the opposite sign. Since $\cosh \left(U_{1}\right)$ is always positive, the sign of the first term hinges on the expression $\tanh \left(U_{1}\right)-x$. Because $d p / d t=0$ in equilibrium, this expression is reduced to $\tanh \left(\alpha_{1} x\right)-x$.

For $\alpha_{1}<1, \tanh \left(\alpha_{1} s\right)-x<0$ for $x>0$ and $\tanh \left(\alpha_{1} x\right)-x>0$ for $x<0$. So, $d x / d t<0$ for $\left(x_{+}, p_{+}\right)$and $d x / d t>0$ for $\left(x_{-}, p_{-}\right)$. There is no other stable equilibria other than fundamental equilibrium.

For $\alpha_{1}>1$, the equation $\tanh \left(\alpha_{1} x\right)-x=0$ has three solutions $(-\bar{x}, 0, \bar{x})$ and

$$
\tanh \left(\alpha_{1}\right)-x\left\{\begin{array}{lll}
>0 & \text { for } & x<-\bar{x} \\
<0 & \text { for } & -\bar{x}<x<0 \\
>0 & \text { for } & 0<x<\bar{x} \\
<0 & \text { for } & x>\bar{x}
\end{array}\right.
$$

Therefore, for $-\bar{x}<x<0$ and $0<x<\bar{x}$, the first and second term in $d x / d t$ carry opposite signs and there could indeed exist additional equilibria $E_{+}\left(x_{+}>\right.$ 
$\left.0, p_{+}>p_{f}\right)$ and $E_{-}\left(x_{-}<0, p_{-}<p_{f}\right)$ where $x_{-}=-x_{+}, p_{+}-f_{f}=p_{f}-p_{-}$and $-\bar{x}<x_{-}<0$ and $0<x_{+}<\bar{x}$.

For any pair $\left(x_{+}, p_{+}\right)$and $\left(x_{-}, p_{-}\right)$, the first term of $d y / d t$ equation is positive. Then $d y / d t=0$ implies $a b(1-y)-a(1-b) y<0$, we can get $y>b$.

In such equilibria, since price $p$ is constant, there is no price-chasing incentive. But the market confidence index $x \neq 0$, there is a force of mimetic to make price far away from fundamental value. It is balanced by the force of fundamentalists who buy (or sell) when price is lower (or higher) than fundamental value which can be inferred from $y>b$.

Proposition 4 presents the symmetry of the possible non-fundamental equilibria.

Proposition 4. Let $E_{1}\left(x_{1}, y_{1}, p_{1}\right)$ be an equilibrium of system (10), then there exists a point $E_{2}\left(x_{2}=-x_{1}, y_{2}=y_{1}, p_{2}=2 p_{f}-p_{1}\right)$ is also an equilibrium.

Proof. With $E_{1}\left(x_{1}, y_{1}, p_{1}\right)$ be an equilibrium of system (10), $\left.\frac{d x}{d t}\right|_{x 1, y 1, p 1}=0,\left.\frac{d y}{d t}\right|_{x 1, y 1, p 1}=$ $0,\left.\frac{d p}{d t}\right|_{x 1, y 1, p 1}=0$.

From $x_{2}=-x_{1}, y_{2}=y_{1}, p_{2}=2 p_{f}-p_{1}$, one gets $U_{1}\left(x_{2}, y_{2}, p_{2}\right)=-\alpha_{1} x_{1}=$ $-U_{1}, U_{2,1}\left(x_{2}, y_{2}, p_{2}\right)=U_{2,2}\left(x_{2}, y_{2}, p_{2}\right)=-s \alpha_{3}\left|\frac{p_{1}-p_{f}}{2 p_{f}-p_{1}}\right|$.

So, $\left.\frac{d x}{d t}\right|_{x 2, y 2, p 2}=2 v_{1}(1-a)\left(1-y_{1}\right)\left[-\tanh \left(U_{1}\right)+x_{1}\right] \cosh \left(U_{1}\right)=-\left.\frac{d x}{d t}\right|_{x 1, y 1, p 1}=$ $0,\left.\frac{d y}{d t}\right|_{x 2, y 2, p 2}=-v_{2}(1-a) y_{1}\left(1-y_{1}\right)\left[\sinh \left(U_{2,1}\right)+\sinh \left(U_{2,2}\right)\right]-a(1-b) y_{1}+$ $a b\left(1-y_{1}\right)=\left.\frac{d y}{d t}\right|_{x 1, y 1, p 1}=0,\left.\frac{d p}{d t}\right|_{x 2, y 2, p 2}=-\beta\left[x_{1}\left(1-y_{1}\right) T_{c}+y_{1}\left(p_{f}-f_{1}\right) T_{f}\right]=$ $-\left.\frac{d p}{d t}\right|_{x 1, y 1, p 1}=0$.

This proposition ensures that if the non-fundamental equilibria exist they must appear in pair and be symmetric to each other by $x=0, p=p_{f}$. Numerical experiments using 4-order Runge-Kutta approach to integrate this ordinary differential equations system confirm these results. In most settings of parameters with $\alpha_{1}>1$, non-fundamental equilibria occur. For example, when $\alpha_{1}=1.5, \alpha_{2}=0.25, \alpha_{3}=1.5$, we get two stable non-fundamental equilibria at $\left(x^{*}=0.85, y^{*}=0.78, p^{*}=10.23\right)$ and $\left(x^{*}=-0.85, y^{*}=0.78, p^{*}=9.77\right)$. We should notice that it does not mean that stable non-fundamental equilibria exist for all $\alpha_{1}>1$, and we will come across exceptions near $\alpha_{1}=1$ in the following part.

\subsection{Periodicity and chaos regimes}

Because of the highly non-linearity of the system, we rely heavily on numerical experiments to investigate the its dynamics. By plenty of Runge-Kutta integration we find that between the ranges of parameters of fundamental equilibrium and non-fundamental equilibria, some complex behaviors such as periodicity and chaos appear. Figure 2 is some numerical experiment results of system (10) with $\alpha_{1}=0.95, \alpha_{2}=0.2, \alpha_{3}=0.5$ where the market is in a periodicity regimes. 

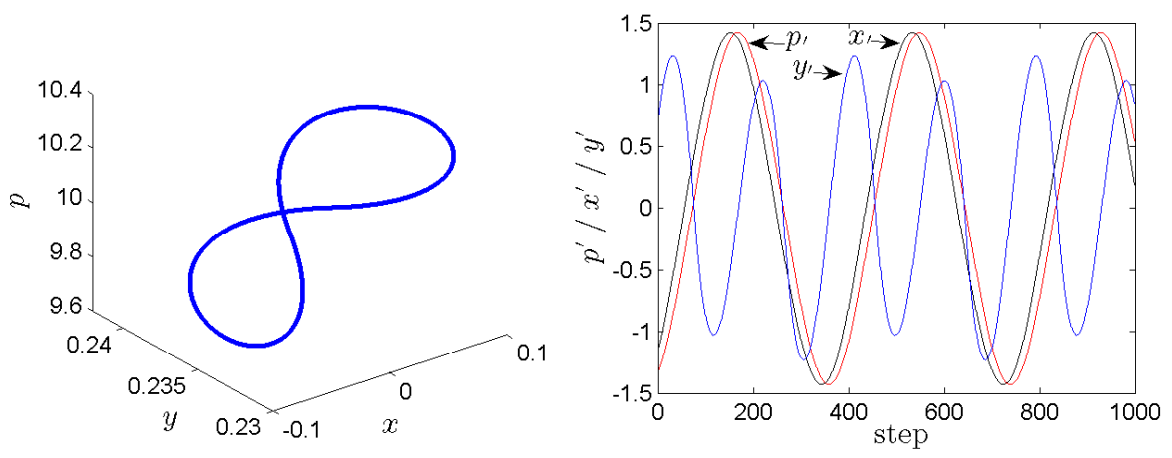

Fig. 2. Numerical experiments of cycle regime

From the stable periodic orbit in the left part of figure 2 we can notice the bull and bear cycle in market evolvement. Such a regime enables us to investigate the check and balance of different forces thoroughly. The right part of figure 2 shows the typical trajectories of $x, y, p$ over a time span after the transitional stage, $x, y, p$ are rescaled as $x^{\prime}=\frac{x-\bar{x}}{\sigma_{x}}$ (black), $y^{\prime}=\frac{y-\bar{y}}{\sigma_{y}}$ (blue), $p^{\prime}=\frac{p-\bar{p}}{\sigma_{p}}$ (red) for visual convenience. In the bull stage, price $p$ is increasing, so the optimistic chartists who buy stocks can earn more, and attract fundamentalists and pessimistic chartists to switch to optimistic chartists, hence $x$ becomes larger and $y$ smaller. Meanwhile, those transitions strengthen the call-side force and make the price $p$ climbe higher. But when there is too much optimistic chartists in market, the number of agents switching to optimistic chartists decreases, that is $d x / d t<0$. At the same time, the deviation of price to fundamental value provides incentives for fundamentalists to arbitrage, so the rationality index $y$ start to fall down first. And the increase of put-side force makes the up-climbing of price become slower and turn down at last. Then the market enters the bear stage.

Figure 3 gives the numerical experiment results of system (10) with $\alpha_{1}=$ $1.008, \alpha_{2}=0.2, \alpha_{3}=0.5$ where the market is in a chaos regimes. The left part is the strange attractor in phase portrait. Investigate the time series of $x, y, p$ (the right part of figure 3 , rescaled as above) there are similar patterns to periodic regime, but balance is broken and these patterns cannot persist for a long time. we can realize that the underlying forces driving the market dynamics is the same, but because all the equilibria and periodic orbit lose stability and the evolution of market exhibits a deterministic randomness.

\subsection{Overall view of regimes with a bifurcation diagram}

In this part, we present a overall view of four different dynamic regimes with a bifurcation diagram, as in figure 4 . We use $\alpha_{1}$ as the control parameter with $\alpha_{2}=0.2$ and $\alpha_{3}=0.5$. For each value of $\alpha_{1}$ from 0 to 2 , we integrate system (10) by 4 -order Runge-Kutta method with a random initial value in a feasible range, say $x \in[-1,1], y \in[0,1], p \in[5,15]$. The objective of choosing random 

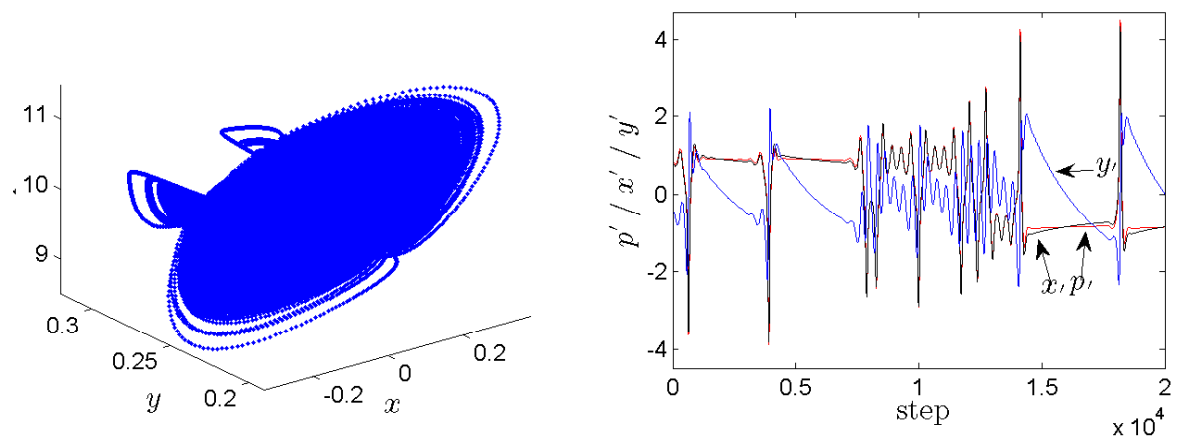

Fig. 3. Numerical experiments of chaos regime

initial value is to find the possibility of coexisting attractors such as coexisting non-fundamental equilibria after $\alpha_{1}=1$, although for a certain $\alpha_{1}$, we integrate once and get one attractor, but by randomness of initial value, the result with $\alpha_{1}$ nearby may come to the other different attractors. After we get the price series by integration, we generalize the idea of first return map introduced in [20] to draw this diagram. The first-return is the successive maximum of the time series for a single variable. Here we find both the local maxima and local minima of price $p$. Because we have to cope with all kinds of regimes, we first judge the solution type, if it is a stable equilibrium, we draw $p$ of this equilibrium versus $\alpha_{1}$ with red color, otherwise, we plot all local maxima and minima of $p$ with blue color.

One may notice that when $\alpha_{1}$ is less than 0.2 approximatively, the conditions in (11) are satisfied, and system (10) is in a fundamental regime(red color). As $\alpha_{1}$ increases, the conditions in (11) are violated, so the fundamental equilibrium loses stability, and a hopf bifurcation occurs with stock market falling into a periodic regime. In the periodic regime, the maximum $p$ is larger than $p_{f}$ and minimum $p$ less than fundamental value $p_{f}$, and the maximum distance from fundamental value gets larger with $\alpha_{1}$ increasing(blue color). When $\alpha_{1}$ is beyond 1 , two symmetric stable non-fundamental equilibria coexist with price lager and smaller than fundamental value respectively, as proposition 3 and 4 indicate. In a narrow range near $\alpha_{1}=1$ chaos appears as illustrated in figure 4 where many points correspond to a single $\alpha_{1}$ value, and the numerical simulation results of this chaotic parameter setting is in figure 3 . All the results coincide with previous analysis.

\section{Conclusion}

This paper presents a multi-agent model with stochastic transition mechanism. The dynamic of the market can be governed by a system of differential equations in three state variables of market confidence index, rationality index and price. 


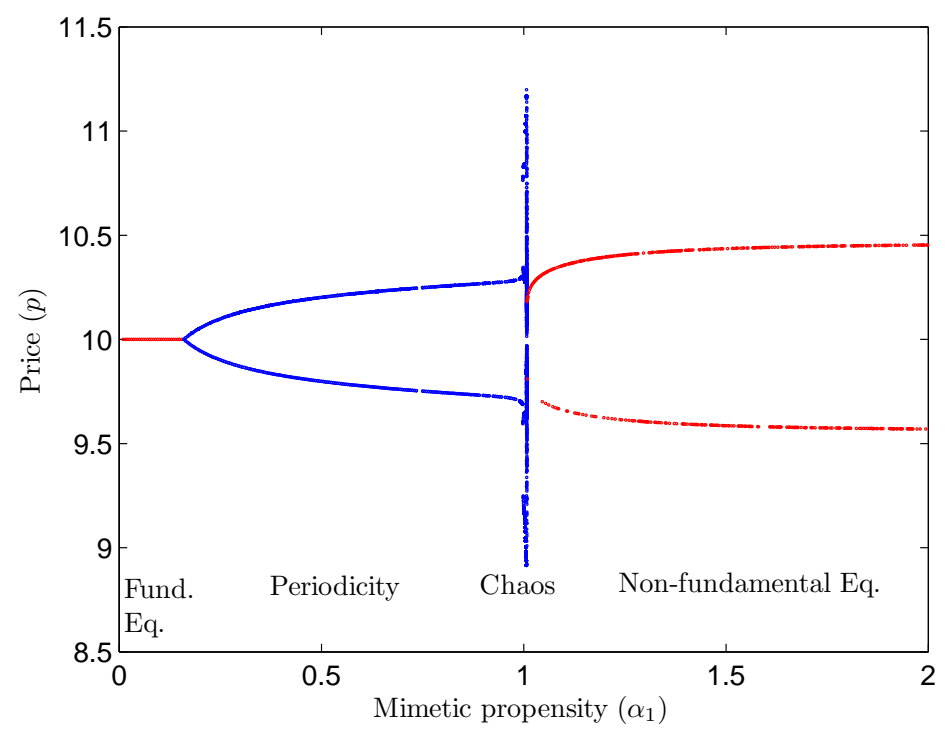

Fig. 4. Bifurcation diagram with $\alpha_{2}=0.2$ and $\alpha_{3}=0.5$

Analysis on this system shows that under different settings of parameters representing traders' propensities, market exhibits four dynamic regimes including fundamental equilibrium, non-fundamental equilibrium, periodicity and chaos. When strategy-switching propensity, price-chasing propensity and mimetic propensity are all small, market is in a fundamental equilibrium; when the stable conditions are violated, a hopf bifurcation leads to periodicity regime. When the mimetic propensity is large enough, two symmetric non-fundamental equilibria coexist. In a narrow range between non-fundamental equilibria and periodicity regime, chaos appears as a transitional phenomena.

Relative to the volatile market price, traders' propensities are much stable, so understanding the influence of traders' propensities to market dynamic regimes may shed light on the complex behavior of the stock market.

The parameter calibration to make the model precise enough to describe the dynamics of a real market is the direction of our further research.

\section{Acknowledgments}

This work was supported by the National Science Foundation of China under Grant No.70871013 and the Program for New Century Excellent Talents in University under Grant No. NCET-07-0089. 


\section{References}

1. Fama, E. F. : Efficient Capital Markets: A Review of Empirical Work. J. Financ. 2, 383-417. (1970)

2. Zhong, M., Darrat, A. F., Anderson, D. C. : Do U.S. stock prices deviate from their fundamental values? Some new evidence. J. Bank. Financ. 27, 673-697 (2003)

3. Sarno, L., Taylor, M. P. : An empirical investigation of asset price bubbles in Latin American emerging financial markets. Appl. Fin. Econ. 13, 635-643 (2003)

4. Brooks C., Katsaris A. : Rational speculative bubbles: an empirical investigation of the London Stock Exchange. Bull. Econ. Res. 55(4), 319-346 (2003)

5. Gonzalez, L., Powell, J. G., Shi, J. A. : Two centuries of bull and bear market cycles. Int. Rev. Econ. Fin. 14, 469-486 (2005)

6. Pagan, A. R., Sossounov, K. A. : A simple framework for analysing bull and bear markets. J. Appl. Econom. 18, 23-46 (2003)

7. Yan, W., Powell, J. G. : Chinese stock market cyclical regimes: 1991-2006. Econ. Lett. 97(3), 235-239 (2007)

8. Peters, E. E. : Chaos and Order in the Capital Markets. Wiley, New York (1991)

9. Chiarella, C. : The dynamics of speculative behaviour. Ann. Oper. Res. 37, 101-23 (1992)

10. Brock, W. A., Hommes, C. H. : A rational route to randomness. Econometrica. 65, 1059-1095 (1997)

11. Brock, W. A., Hommes, C. H. : Heterogeneous beliefs and bifurcation routes to chaos in a simple asset pricing model. J. Econ. Dyn. Control. 22, 1235-1274 (1998)

12. Hommes, C. H. : Financial markets as nonlinear adaptive evolutionary systems. Quant. Fin. 1, 149-67 (2001)

13. Chiarella, C., He, X. : Asset pricing and wealth dynamics under heterogeneous expectations. Quant. Fin. 1, 509-526 (2001)

14. Chiarella, C., Dieci, R., Gardini, L. : Speculative behaviour and complex asset price dynamics. J. Econ. Behav. Organ. 49, 17-97 (2004)

15. Shefrin, H. : Beyond Greed and Fear. Oxford University Press, New York (2002)

16. Shiller, J. : From Efficient Markets Theory to Behavioral Finance. J. Econ. Perspect. 17, 83-104 (2003)

17. Lux, T., Marchesi, M. : Scaling and criticality in a stochastic multi-agent model of a financial market. Nature. 397, 498-500 (1999)

18. Lux, T. : The socio-economic dynamics of speculative markets: interacting agents, chaos, and the fat tails of return distributions. J. Econ. Behav. Organ. 33, 143-165 (1998)

19. Lux T. : Herd Behaviour, Bubbles and Crashes. Econ. J. 105, 881-896(1995)

20. Lorenz, N. : Deterministic non-periodic flow. J. Atmos. Sci. 20, 130-141 (1963) 